\title{
Analyzing time evolution of constraint equations of Einstein's equation
}

\author{
Ryosuke Urakawa $^{1}$, Takuya Tsuchiya ${ }^{2}$ and Gen Yoneda ${ }^{2}$ \\ ${ }^{1}$ Global Education Center, Waseda University, 1-104 Totsuka-cho, Shinjuku-ku, Tokyo 169- \\ 8050, Japan \\ ${ }^{2}$ Department of Mathematics, Faculty of Science and Engineering, Waseda University, 3-4-1 \\ Okubo, Shinjuku-ku, Tokyo 169-8555, Japan \\ E-mailurakawa_ryo@aoni.waseda.jp
}

Received October 27, 2018, Accepted December 20, 2018

\begin{abstract}
We propose a method of analyzing the time evolution of constraint equations of the Einstein's equation on the nonflat background by adding constraint terms to the evolution equations. In past studies, the eigenvalues of the coefficient matrix of the constraint equations was mostly obtained on the flat background. Since simulations do not always perform near the flat background, we need to calculate the eigenvalues on the appropriate background. We analyze eigenvalues numerically and predict the stability in the appropriate background. We also perform some numerical simulations and show consistency between the results of the eigenvalue analysis and the numerical stability.
\end{abstract}

Keywords Einstein's equation, numerical relativity, eigenvalue, numerical stability

Research Activity Group Scientific Computation and Numerical Analysis

\section{Introduction}

Numerical relativity is one of the fields in general relativity to solve the Einstein's equation numerically. One of the roles of numerical relativity is to make waveform templates for gravitational observatories. So far, gravitational waves have been observed six times by the advanced LIGO and the advanced Virgo [1]. The six gravitational wave events appear to have originated from the merger of two black holes or two neutron stars. These data were compared with waveform templates obtaining by numerical simulations. Numerical relativity is hereafter expected to attract considerable attentions owing to its importance for future gravitational wave events. Since more precise numerical results must be required in the future, it is desirable to improve the numerical techniques. Up to the present, various improvements have been reported and used. One of the most basic formulations of the Einstein equations is the ArnowittDesser-Misner (ADM) formulation [2-4]. Nowadays, the Baumgarte-Shapiro-Shibata-Nakamura (BSSN) formulation $[5,6]$ based on the ADM formulation is widely used to perform black hole merger simulations. In this Letter, we treat the ADM formulation.

Some authors have studied some numerical techniques for performing precise simulations using the evolution equations of constraints [7-12]. The equations are called the constraint propagation equations. We can predict the numerical stability of the system with constraints in advance by analyzing them. Concretely, we calculate the eigenvalues of the coefficient matrix of the constraint propagation equations. We refer to these eigenvalues as constraint amplification factors $(\mathrm{CAF})$. In the previous studies [7-9], CAF was mostly calculated on the flat (Minkowski) background (as far as we know, there has only been one study on the Schwarzchild background [10]). Here we use "background" as the exact solution of the Einstein's equation to which numerical solution is close. Although the usefulness of calculating CAF on the Minkowski background has been demonstrated by some simulations [13], the analysis of CAF on the Minkowski background sometimes appears to be insufficient because the background is not always the Minkowski background. Therefore, we should use a background corresponding to each simulation. However, it is difficult to analytically calculate CAF on a nonflat background. In this Letter, we calculate it numerically and compare the numerical stability between the predictions with CAF in the Minkowski background and the one in the background corresponding to the simulation.

This Letter is composed of the following sections. First, we review the decomposition of the Einstein's equations and the method of the numerical calculation in Sec. 2. Next, we review CAF on the Minkowski background, the adjusted formulation and calculate it on a nonflat background in the a Sec. 3. We show the results of our simulations and confirm that the results match the CAF analysis in Sec. 4. Finally, we summarize our results in Sec. 5.

\section{Numerical Calculation of Einstein's equation}

2.1 Evolution equations and constraint equations

Einstein's equation describes the fabric of spacetime and the distribution of matter. This equation is written 


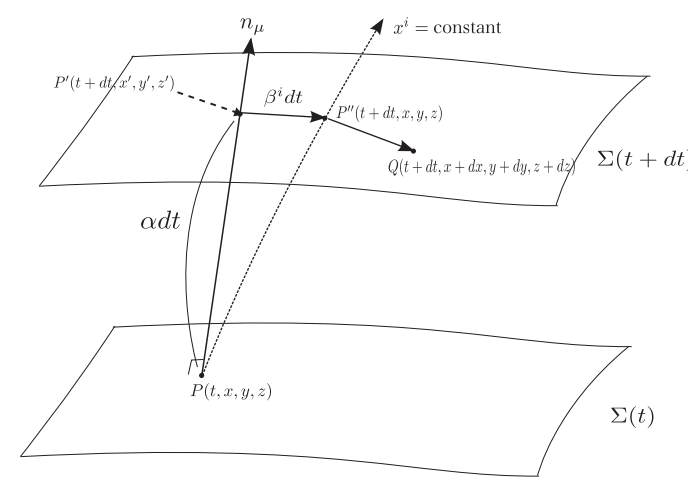

Fig. 1. This figure shows the decomposition of spacetime. $\Sigma(t)$ denotes the spacelike 3-dimensional hypersurface on time $t$. The 4-dimensional spacetime can be foliated by a family of hypersurface. This foliation can split the spacetime into the time and space direction. $n^{\mu}$ is the unit normal to the hypersurface.

by

$$
R_{\mu \nu}-(1 / 2) g_{\mu \nu} R=8 \pi T_{\mu \nu},
$$

where $g_{\mu \nu}$ is the metric, $R_{\mu \nu}$ is the Ricci tensor and $R$ is the Ricci scalar in the spacetime. $T_{\mu \nu}$ is the energymomentum tensor. The Latin indices run from 0 to 3 and the Greek indices run from 1 to 3 . In addition, we use the Einstein summation convection.

Einstein's equation is classified second order nonlinear partial differential equations with respect to the metric $g_{\mu \nu}$. In addition, we cannot calculate numerically as the initial value problem in this original formulation because the time and space treat equivalently and then we cannot decide the time direction. We generally split the spacetime into time and space. We show the concept in Fig. 2.1. The line element $d s^{2}=g_{\mu \nu} d x^{\mu} d x^{\nu}$ is split as

$$
d s^{2}=-\alpha^{2} d t^{2}+\gamma_{i j}\left(d x^{i}+\beta^{i} d t\right)\left(d x^{j}+\beta^{j} d t\right),
$$

where $\gamma_{i j}$ is the induced three-metric, $\alpha \equiv 1 / \sqrt{g^{00}}$ is the lapse function and $\beta_{i} \equiv g_{0 i}$ is the shift vector. These variables represent the deviation of the time and space coordinates associated with the fabric of spacetime. We can split the Einstein's equation into the following evolution equations and the constraint equations.

$$
\begin{aligned}
\partial_{t} \gamma_{i j}=- & 2 \alpha K_{i j}+D_{i} \beta_{j}+D_{j} \beta_{i} \\
\partial_{t} K_{i j}=\alpha & \left({ }^{(3)} R_{i j}+K K_{i j}-2 K_{i k} K_{j}^{k}\right)-D_{i} D_{j} \alpha \\
& +K_{k i} D_{j} \beta^{k}+K_{k j} D_{i} \beta^{k}+\beta^{k} D_{k} K_{i j} \\
& -8 \pi \alpha\left[S_{i j}-(1 / 2) \gamma_{i j}\left(S-\rho_{H}\right)\right],
\end{aligned}
$$

where $K_{i j}$ is the extrinsic curvature. $\left(\gamma_{i j}, K_{i j}\right)$ are the dynamical variables in this formulation. $D_{i}$ is the covariant derivative associated with $\gamma_{i j},{ }^{(3)} R_{i j}$ is the threeRicci tensor, and $K$ is the trace of the extrinsic curvature defined by $K=\gamma^{i j} K_{i j}$. The matter sources are defined by $\rho_{H}=n_{\mu} n_{\nu} T^{\mu \nu}, S_{i j}=\gamma_{\mu i} \gamma_{\nu j} T^{\mu \nu}, S_{i}=-\gamma_{\nu i} n_{\mu} T^{\mu \nu}$ and $S=\gamma^{i j} S_{i j}$ where $n_{\mu}=(-\alpha, 0,0,0)$.

In addition, the constraint equations are

$$
\begin{aligned}
\mathcal{H} & :={ }^{(3)} R+K^{2}-K_{i j} K^{i j}-16 \pi \rho_{H} \approx 0, \\
\mathcal{M}_{i} & :=D_{j} K^{j}{ }_{i}-D_{i} K-8 \pi S_{i} \approx 0,
\end{aligned}
$$

Solve the constraint equations and obtain the initial values.

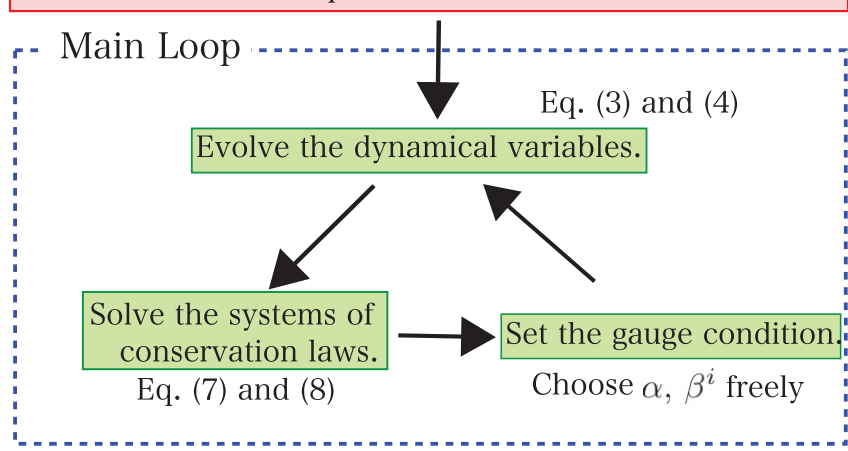

Fig. 2. The flow chart of calculating the Einstein's equation numerically.

where ${ }^{(3)} R$ is the three-scalar curvature defined by ${ }^{(3)} R=\gamma^{i j(3)} R_{i j}$. (5) and (6) are called the Hamiltonian constraint and the momentum constraints, respectively.

\section{2 $3+1$ equations of matter}

In this Letter, we treat the perfect fluid with no pressure. That is, $T^{\mu \nu}=\rho u^{\mu} u^{\nu}$ where $\rho$ denotes the mass energy density and $u^{\mu}$ denotes the 4 -velocity. The evolution equations with respect to the $\left(\rho, u_{i}\right)$ are derived by the decomposition of the energy conservation law $\nabla_{\mu} T_{\nu}^{\mu}=0$. The evolution equations are written as follows.

$$
\begin{aligned}
& \partial_{t} \rho_{*}+\partial_{i}\left(\rho_{*} v^{i}\right)=0 \\
& \partial_{t}\left(\rho_{*} u_{i}\right)+\partial_{j} \rho_{*} v^{j} u_{i} \\
& \quad=\rho_{*}\left(\alpha u^{0} \partial_{i} \alpha-u_{i} \partial_{j} \beta^{j}+\frac{1}{2 u^{0}} u_{k} u_{l} \partial_{i} \gamma^{k l}\right),
\end{aligned}
$$

where $\rho_{*} \equiv \rho \alpha u^{0} \sqrt{\gamma}$ (the static mass density) and $v^{i} \equiv u^{i} / u^{0}$. The velocity $u^{0}$ can be decided by the normalization condition $g_{\mu \nu} u^{\mu} u^{\nu}=-1$ and $\gamma$ is the determinant of $\gamma_{i j}$. In the simulation, the dynamical variables are $\left(\rho_{*}, v^{i}\right)$ instead of $\left(\rho, u_{i}\right)$.

\subsection{The method of numerical calculation}

We show the flow chart to solve the Einstein's equation numerically in Fig. 2.3. (i) First, we solve the constraints to construct the initial data $\left(\gamma_{i j}, K_{i j}\right)$. (ii) Next, we evolve the dynamical variables $\left(\gamma_{i j}, K_{i j}\right)$ by using the evolution equations. (iii) In parallel, we determine the dynamics of the fluid by using the evolution of equations with respect to $\left(\rho_{*}, v^{i}\right)$. (iv) Then we determine a gauge (coordinate) condition $\left(\alpha, \beta^{i}\right)$ freely. That is, we can choose the convenient coordinates. (v) We repeat the procedure (i)-(iv) and check the constraints whether or not to preserve. (vi) We stop the numerical calculation if we reach the intended time, or either constraints or dynamical values diverge.

\section{Constraint propagation equations and $\mathrm{CAF}$}

\section{$3.1 C A F$}

To investigate the numerical stability, we introduce the constraint propagation equations which are the evolution equations of constraints. We can predict the be- 
havior of the constraints in advance by analyzing the set of eigenvalues of the coefficient Fourier transformed matrix of the constraint propagation equations. Then we show the details. We suppose the dynamical variables $v^{k}(\vec{x}, t)$ satisfy the evolution equations

$$
\partial_{t} v^{k}=f\left(v^{k}, \partial_{i} v^{k}, \ldots\right),
$$

and the first class constraint equations

$$
C^{l}\left(v^{k}, \partial_{i} v^{k}, \ldots\right) \approx 0
$$

Next, we analytically calculate the constraint propagation equations

$$
\partial_{t} C^{l}=g\left(C^{l}, \partial_{i} C^{l}, \ldots\right) .
$$

Finally, we apply the Fourier transformation to the constraint propagation equations. That is,

$$
\partial_{t} \hat{C}^{l}=g\left(\hat{C}^{l}\right)=M_{m}^{l} \hat{C}^{m},
$$

where $C^{l}(\vec{x}, t)=\int \hat{C}^{l}(\vec{k}, t) \exp (\mathrm{i} \vec{k} \cdot \vec{x}) d^{3} k$, i is the imaginary unit and $\vec{k}$ is the wave vector. We call the eigenvalues of the coefficient matrix $M_{m}^{l}$ the constraint amplification factor (CAF). Negative real parts of CAF imply the constraints will decay, and positive real parts of CAF imply that the constraints will increase. Namely, smaller real parts of CAF implies less rapid growth of the constraints. On the other hand, if CAF has non-zero imaginary parts, the constraints almost do not changed. Therefore, we expect more stable simulation in the system that CAF has negative real parts and non-zero imaginary parts.

By using this method, we analyze the behavior of violations of the constraints in the Einstein's equation. The constraint propagation equations of (5) and (6) can be written as follows [7]

$$
\begin{aligned}
& \partial_{t}\left[\begin{array}{c}
\mathcal{H} \\
\mathcal{M}_{i}
\end{array}\right] \\
& =\left[\begin{array}{cc}
\beta^{k} D_{k}+2 \alpha K & -2 \alpha \gamma^{k j} D_{k} \\
-\frac{1}{2} \alpha D_{i}-D_{i} \alpha & \beta^{k} D_{k} \delta^{j}{ }_{i}+D_{i} \beta^{j}+\alpha K \delta^{j}{ }_{i}
\end{array}\right]\left[\begin{array}{c}
\mathcal{H} \\
\mathcal{M}_{j}
\end{array}\right] .
\end{aligned}
$$

From this, the constraint equations always satisfy every time if the initial data is satisfy the constraint equations. Also, the equations do not change under any matter fields [14].

In the Minkowski background, we can obtain CAF by substituting $\alpha=1, \beta^{i}=(0,0,0), \gamma_{i j}=\operatorname{diag}(1,1,1)$, $K_{i j}=\operatorname{diag}(0,0,0)$ and change $D_{i}$ into $\mathrm{i} \vec{k}$. This change means the Fourier transformation. In this Letter, we treat the wave vector as $\vec{k}=(1,0,0)$. Then, the CAF is $(0,0, \mathrm{i},-\mathrm{i})$ on this system in the Minkowski background. Therefore, the system will stop because of the low frequency error growing modes.

\subsection{Adjusted formulation}

We briefly review the idea of the adjusted formulation to enable calculating stable simulations [7-9]. Fundamentally, we rewrite the evolution equations by adding the constraints to them so that the constraints can decay. If the adjusted terms are added to the evolution equations, then the constraint propagation equations and the
CAF will change. Therefore, we conclude that the adjustment is effective if it leads to smaller real parts of the CAF.

There are various combinations of the adjusted terms. We now add the following the adjusted term to the evolution equation of $K_{i j}$.

$$
\partial_{t} K_{i j}=(\text { right hand side of }(4))+\kappa \alpha \gamma_{i j} \mathcal{H},
$$

where $\kappa$ is a constant. Basically, $|\kappa|$ is set to small because the system keeps the low frequency error-growing modes.

In this adjusted formulation, the constraint propagation equations change a bit as follows

$$
\begin{array}{r}
\partial_{t}\left[\begin{array}{c}
\mathcal{H} \\
\mathcal{M}_{i}
\end{array}\right]=(\text { coefficient matrix of }(13))\left[\begin{array}{c}
\mathcal{H} \\
\mathcal{M}_{i}
\end{array}\right] \\
+\left[\begin{array}{cc}
4 \kappa \alpha K & 0 \\
-2 \kappa\left(D_{i} \alpha+\alpha D_{i}\right) & 0
\end{array}\right]\left[\begin{array}{c}
\mathcal{H} \\
\mathcal{M}_{i}
\end{array}\right] .
\end{array}
$$

On this system, the CAF is $(0,0, \mathrm{i} \sqrt{1+4 \kappa},-\mathrm{i} \sqrt{1+4 \kappa})$ in the Minkowski background. If $1+4 \kappa>0$, the stability of the system will not change. On the other hand, If $1+4 \kappa<0$, the CAF has a negative real part and a positive real part. Therefore, the system will possibly get an unstable mode.

In the next section, we investigate CAF in the background of the simulation which is not the Minkowski background, and we perform a simulation for confirmation of the CAF.

\section{Numerical Example}

We show the numerical simulation related to the gravitational collapse to identify the validity of the CAF in the appropriate background, not in the Minkowski background. We assume that the metric is written by

$$
d s^{2}=-d t^{2}+f(t, r) d r^{2}+g(t, r)\left(d \theta^{2}+\sin ^{2} \theta d \phi^{2}\right),
$$

in the sphere polar coordinate where $f(t, r), g(t, r)$ are sufficient smooth functions with respect to $t$ and $r$. We will simply take the simulation domain as $r \in[0,3], \theta=$ $\pi / 2$ and $\phi=0$. We prepare the initial data to satisfy the constraint equations. In particular, we take $f(0, r)=1$, the initial mass density $\rho=0.001 \exp \left[-(r-2)^{2}\right]$ and the initial 4-velocity $u^{\mu}=\left(u^{0},-0.1 r, 0,0\right)$ and then solve the Hamiltonian equation to evaluate $g(0, r)$.

Fig. 3 and Fig. 4 show the CAF and the L2 norm of the Hamiltonian constraint, respectively. Even though there are four components of the $\mathrm{CAF}$, we take the maximal real parts of them in the $x$-direction because the positive real parts are important factor of the numerical stability. We show the results in Fig. 3 which shows the maximal real parts with respect to $x$ (i.e. $\left.\max _{0 \leq x \leq 2 \pi} \max \left(\operatorname{Re}\left(\Lambda_{1}, \Lambda_{2}, \Lambda_{3}, \Lambda_{4}\right)\right)\right)$ where $\Lambda_{i}(i=$ $1,2,3,4)$ are the components of the CAF. We denote this maximum value as $\max (\operatorname{Re}(\mathrm{CAF}))$. Fig. 3 indicates that $\max (\operatorname{Re}(\mathrm{CAF}))$ is smaller if we choose around $\kappa=-0.25$. From Fig. 3, we can predict that the simulation will be more stable if we set $\kappa$ to the negative value which is predicted by the CAF than the positive value or zero. Actually, Fig. 4 implies that the simulation of the negative $\kappa$ case is more better because the constraint 


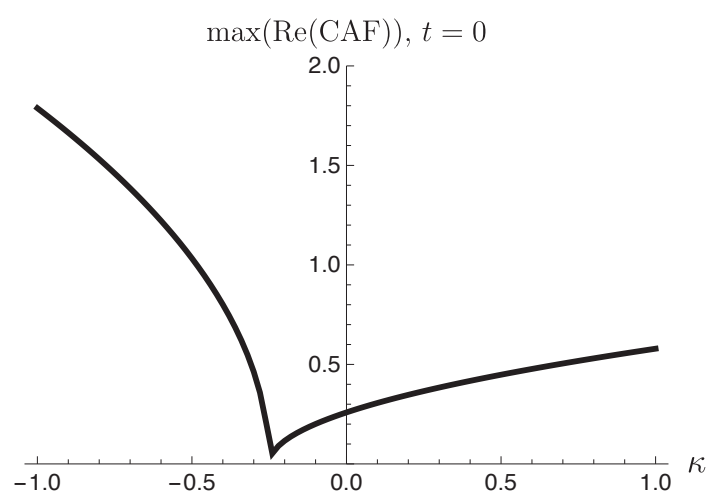

Fig. 3. The maximal real parts of the CAF with respect to $x$ on the background corresponding to the simulation. The horizontal axis indicates $\kappa$ and the vertical axis indicates the maximal real part of each the CAF with respect to $x$. The graph shows that the simulation will be more stable if $\kappa$ is chosen around -0.25 .

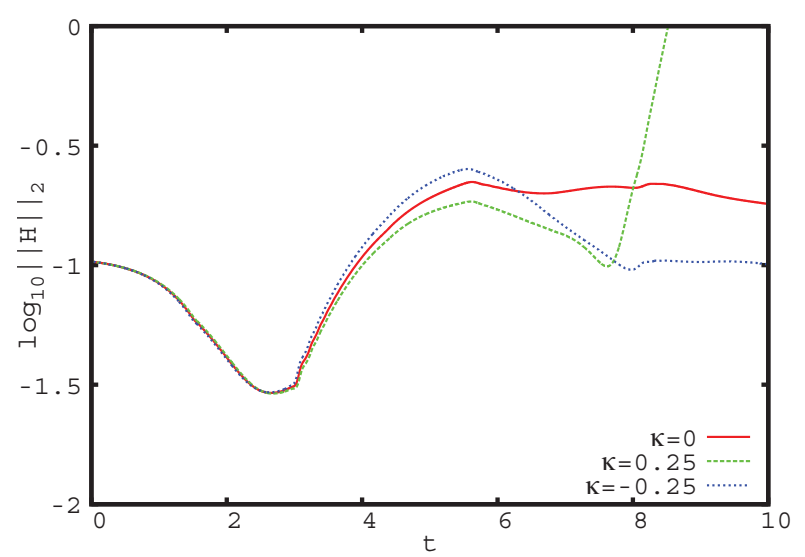

Fig. 4. L2 norm of the Hamiltonian constraint in the simulation. The horizontal axis indicates time and the vertical axis indicates the logarithm of the L2 norm of the Hamiltonian constraint, $\log _{10}\|\mathcal{H}\|_{2}$. The violations of Hamiltonian constraint of $\kappa=$ -0.5 are smaller than others.

decays. Therefore, we can investigate the numerical stability more precisely in our method than the Minkowski background case.

\section{Summary}

We proposed a method of analyzing CAF on a nonflat background based on an adjusted formulation. Concretely, we presented an adjusted formulation and calculated the CAF on the Minkowski background and the gravitational collapse case, and performed some simulations of the gravitational collapse. As a result, we found the consistency between the numerical simulations and the predictions by CAF on the appropriate backgrounds, and the inconsistency on the Minkowski background. On the basis of our results, we believe that it is necessary to use the background corresponding to each simulation when calculating CAF.

Our method can be used with or without the existence of the analytical background. Concretely, when it is difficult to find the analytic background for a simulation, we can use the numerical metric as the background to calculate CAF. Consequently, we strongly believe that our method is valid and will be useful for simulating actual physical models.

\section{Acknowledgements}

Gen Yoneda was partially supported by a Waseda University Grant for Special Research Projects (number 2018-K200, 2018-B102).

\section{References}

[1] B. P. Abbott et al. (LIGO Scientific Collaboration and Virgo Collaboration), Observation of Gravitational Waves from a Binary Neutron Star Inspiral, Phys. Rev. Lett., 119 (2017), 161101.

[2] R. Arnowitt, S. Deser and C. W. Misner, Gravitation: An Introduction to Current Research, Wiley, New York, 1962.

[3] J. W. York, Jr., Sources of Gravitational Radiation, Cambridge University Press, Cambridge, 1979.

[4] L. Smarr and J. W. York, Jr., Kinematical conditions in the construction of spacetime, Phys. Rev. D, 17 (1978), 25292551.

[5] M. Shibata and T. Nakamura, Evolution of threedimensional gravitational waves: Harmonic slicing case, Phys. Rev. D, 52 (1995), 5428-5444.

[6] T. W. Baumgarte and S. L. Shapiro, Numerical integration of Einstein's field equations, Phys. Rev. D, 59 (1998), 024007.

[7] G. Yoneda and H. Shinkai, Hyperbolic formulations and numerical relativity: II. asymptotically constrained systems of Einstein equations, Classical Quantum Gravity, 18 (2001), 441-462.

[8] G. Yoneda and H. Shinkai, Constraint propagation in the family of ADM systems, Phys. Rev. D, 63 (2001), 124019.

[9] G. Yoneda and H. Shinkai, Advantages of a modified ADM formulation: Constraint propagation analysis of the Baumgarte-Shapiro-Shibata-Nakamura system, Phys. Rev. D, 66 (2002), 124003.

[10] H. Shinkai, and G. Yoneda, Adjusted ADM systems and their expected stability properties: constraint propagation analysis in Schwarzschild spacetime, Classical Quantum Gravity, 19 (2002), 1027-1049.

[11] T. Tsuchiya, G. Yoneda, and H. Shinkai, Constraint Propagation of $C^{2}$-adjusted formulation, Phys. Rev. D, 83 (2011), 064032 .

[12] T. Tsuchiya, G. Yoneda, and H. Shinkai, Constraint Propagation of $C^{2}$-adjusted Formulation II - Another Recipe for Robust BSSN Evolution System, Phys. Rev. D, 85 (2012), 044018.

[13] H. Yo, Z. Cao, C. Lin and H. Pan, Improved fast-rotating black hole evolution simulations with modified BaumgarteShapiro-Shibata-Nakamura formulation, Phys. Rev. D, 92 (2015), 024034.

[14] H. Shinkai and G. Yoneda, Constraint propagation in N+1 dimensional space-time, Gen. Relativity Gravitation, 36 (2004), 1931-1937. 